\title{
On degenerate Kirchhoff-type problems with unbounded initial data
}

\author{
Haroldo Clark ${ }^{1}$ and Ronald Guardia ${ }^{2}$ \\ ${ }^{1}$ Universidade Federal do Delta do Parnaíba \\ ${ }^{2}$ UERJ
}

October 4, 2021

\begin{abstract}
This paper deals with the existence, uniqueness and stability uniform of nonlocal solutions for initial-boundary value problems of the Kirchhoff type. The main purpose is to establish the exis- tence of at least one nonlocal solutions for degenerate Kirchhoff-type problems with initial data in the Sobolev spaces and without any restrictions on the size of their norms.
\end{abstract}

\section{Hosted file}

Kirchhoffeq_Geometric_Vfinal_2021_Clark_Guardia.pdf available at https://authorea.com/users/ 439301/articles/540294-on-degenerate-kirchhoff-type-problems-with-unbounded-initial-data 